\title{
Organization of the Visual Cortex in Human Albinism
}

\author{
Michael B. Hoffmann, ${ }^{1}$ David J. Tolhurst, ${ }^{2}$ Anthony T. Moore, ${ }^{3}$ and Antony B. Morland ${ }^{1}$ \\ ${ }^{1}$ Department of Psychology, Royal Holloway, University of London, Egham, Surrey TW20 OEX, United Kingdom, ${ }^{2}$ Department of Physiology, University of \\ Cambridge, Cambridge CB2 3EG, United Kingdom, and ${ }^{3}$ Institute of Ophthalmology, University College, London EC1V 9EL, United Kingdom
}

In albinism there is an abnormal projection of part of the temporal retina to the visual cortex contralateral to the eye. This projection, together with the normally routed fibers from nasal retina, provides a cortical hemisphere with visual input from more than the normal hemifield of visual space. In many mammalian models of albinism, a possible sensory mismatch in the visual cortex is avoided either by reorganization of the thalamocortical connections to give the abnormal input an exclusive cortical representation, or by the abnormal input being substantially suppressed. In this study we examine, with fMRI, how the human visual cortex topographically maps its input in albinism. We find that the input from temporal retina is not substantially suppressed and forms a retinotopic mapping that is superimposed on the mapping of the nasal retina in striate and extrastriate areas. The abnormal routing of temporal fibers is not total, with the line of decussation shifting to between 6 and $14^{\circ}$ into temporal retina. Our results indicate that the abnormal input to visual cortex in human albinism does not undergo topographic reorganization between the thalamus and cortex. Furthermore, the abnormal input is not significantly suppressed in either striate or extrastriate areas. The topographic mapping that we report in human does not conform, therefore, to the commonly observed patterns in other mammals but takes the form of the "true albino" pattern that has been reported rarely in cat and in the only other individual primate studied.

Key words: albino; decussation; chiasm; visual cortex; visual areas; retinotopic mapping; fMRI; human

\section{Introduction}

Albinism has a profound effect on the development of the visual system and pathways. Normally, visual fibers from the human nasal retina cross the midline and project to the hemisphere contralateral to the eye, whereas temporal retinal fibers remain uncrossed and project to the hemisphere ipsilateral to the eye. The line of decussation that normally divides crossed from uncrossed fibers coincides therefore with the vertical meridian through the fovea. In striate cortex, the normal visual representation is a highly organized retinotopic map of the contralateral visual field. Information from corresponding retinal locations of the different eyes is initially segregated into neighboring ocular dominance columns but converges within striate and extrastriate areas to give rise to binocular visual function. The normal projection of visual fibers from the retina is severely disrupted in albinism, where the line of decussation is shifted into the temporal retina, such that a far greater number of fibers from temporal retina cross the midline and project contralaterally than in the normal (see Fig. 2) (Lund, 1965; Creel, 1971; Guillery and Kaas, 1973; Creel et al., 1974; Guillery et al., 1975; Apkarian et al., 1983; Guillery, 1986; Hedera et al., 1994; Jeffery, 1997; Morland et al., 2001, 2002). Studying cortical visuotopy in albinism, therefore,

Received March 24, 2003; revised Aug. 11, 2003; accepted Aug. 11, 2003.

This work was supported by the Wellcome Trust (Grant 063343). M.B.H. acknowledges with thanks support by the Deutsche Forschungsgemeinschaft ( $\mathrm{HO}-2002-3)$ and the Wellcome Trust. We are particularly grateful for the cooperation of the subjects. We thank the members of the Radiological Sciences Unit at the Charing Cross Hospital for their assistance. We also are indebted to Dr. Gavin Houston and Elisabeth von dem Hagen, Royal Holloway, for their assistance with data collection.

Correspondence should be addressed to Antony B. Morland, Department of Psychology, Royal Holloway, University of London, Egham, Surrey TW20 OEX, UK. E-mail: a.morland@rhul.ac.uk.

Copyright $\odot 2003$ Society for Neuroscience $\quad 0270-6474 / 03 / 238921-10 \$ 15.00 / 0$ offers a unique opportunity to establish how the human visual cortex can topographically distribute an abnormal retinal input.

No data are available on the organization of the visual cortex of humans with albinism, but studies in animal models of albinism indicate varying degrees of reorganization of the normal cortical topographic representation as reviewed in Table 1. The geniculostriate projection can be reordered (the "Boston" pattern); as a consequence, the abnormal visual input from the thalamus is given an exclusive cortical representation that is contiguous with the normal retinotopic map as found in some Siamese cats (Hubel and Wiesel, 1971) and also in albino ferrets (Huang and Guillery, 1985; Ackerman et al., 2003). Other studies have indicated an absence of any reordering of the geniculostriate projection (the "Midwestern" pattern); however, these studies also document that very few cortical cells appear to respond to stimuli presented to retinal locations from where fibers are misrouted to the cortex (Kaas and Guillery, 1973). This has been taken as evidence of intracortical suppression and is consistent with a lack of behavioral sensitivity to stimulation of the temporal retina in some hypopigmented cats (Elekessy et al., 1973; Guillery et al., 1974) and ferrets (Garipis and Hoffmann, 2003). The extent of intracortical suppression has also been found to vary in its strength (Guillery et al., 1984; Leventhal and Creel, 1985), because there are also reports of robust cortical responses to stimulation of the temporal retina in an albino monkey and some truly albino cats (the "true albino" pattern). This indicates that an absence of geniculostriate reordering does not always lead to a total suppression of cortical responses. It should be noted that some studies demonstrate the existence of both Boston and Midwestern patterns within area 17 of the same animal at exclusive cortical regions (Cooper and Blasdel, 1980; Berman and Grant, 
1992; Ackerman et al., 2003). It appears, therefore, that the observed pattern of organization depends on the locations of electrode penetrations in visual cortex, which could question the interpretations of electrophysiological studies that were restricted to a small number of recording sites.

To date, the visuotopic map in the cortex of albino primates has been studied in detail in only one non-human subject (Guillery et al., 1984), which showed the true albino pattern, the least common of the patterns expressed in animal models of albinism (Table 1). We have examined the visual cortical responses of four humans with albinism, using a functional magnetic resonance imaging (fMRI) retinotopic mapping procedure (Engel et al., 1997). This method offers the advantage over electrophysiological techniques of sampling signals from a large contiguous region of cortical tissue. Our results provide evidence for a true albino pattern of organization in striate cortex of all our subjects with albinism, indicating that the geniculostriate projections were not reordered. Superimposed mirror-symmetrical representations of the nasal and temporal retina were demonstrated not only in striate cortex, but also in extrastriate visual areas, which indicates that the abnormal input is used in areas beyond V1.

\section{Materials and Methods}

Subjects. Two female controls, aged 22 and 21 years, with no neurological or ophthalmological history and normal visual acuity, and four female subjects with albinism, A1, A2, A3, and A4, aged 23-32 years, were the subjects for the experiments. All subjects gave their informed written consent. The study had approval from the Riverside Ethics Committee and the Royal Holloway Ethics Committee. The four subjects with albinism showed the typical features of albinism (Oetting et al., 1996). Their visual evoked potentials showed lateralization that is typical of chiasmal misrouting seen in all forms of albinism (Apkarian et al., 1983). A1 and A2 were unusual in having minimal nystagmus in the primary position; A3 and A4 did have nystagmus. The left eyes of the control subjects and of $\mathrm{A} 1, \mathrm{~A} 2$, and $\mathrm{A} 3$ and the right eye of A4 were stimulated during the retinotopic mapping experiments. The visual acuity of the eye stimulated in the fMRI experiments was measured with the Freiburg visual acuity test (Bach, 1996). In A1, A2, A3, and A4, visual acuity was 0.5, 0.5, 0.2, and 0.2 , respectively. Peak to peak nystagmus amplitude was also determined outside the scanner with values of $1.5,1.5,3.0$, and $3.0^{\circ}$ for $\mathrm{A} 1, \mathrm{~A} 2$, $\mathrm{A} 3$, and A4, respectively. It has been shown previously that fixation instabilities of moderate extent have only little effect on the fMRI response (Baseler et al., 2002).

Visual stimulation-fMRI measurements. The stimulus patterns were based on a checkerboard consisting of black and white checks that were each bounded by concentric circles $1.9^{\circ}$ of visual angle apart and by radii angled at $15^{\circ}$ to each other ("circular checkerboard"). The center of the pattern was fixated, and its overall diameter was $45^{\circ}$ of visual angle. There were thus 24 segments and 12 rings of alternating black and white checks. The mean luminance was $35 \mathrm{~cd} / \mathrm{m}^{2}$, and the contrast of the checks was $90 \%$. The black and white checks reversed polarity at $6 \mathrm{~Hz}$. The entire stimulus was spanned by a red cross, which served as a highly salient fixation target suitable for subjects with low visual acuity.

For retinotopic mapping (Sereno et al., 1995; DeYoe et al., 1996; Engel

Table 1. Summary of previous reports on the cortical organization pattern in animal models of albinism $(n / m ;$ where $n=$ number of individuals with a given pattern; $m=$ number of individuals studied)

\begin{tabular}{|c|c|c|c|c|c|c|c|}
\hline \multirow[b]{2}{*}{ Animal } & \multirow[b]{2}{*}{ Study } & \multicolumn{3}{|l|}{ Pattern } & \multicolumn{3}{|c|}{ Method } \\
\hline & & B & M & A & $E$ & H & B \\
\hline \multicolumn{7}{|l|}{ Cat } & \\
\hline Siamese & Hubel and Wiesel, 1971 & $5 / 7$ & $2 / 7$ & & $x$ & & \\
\hline Siamese & Kaas and Guillery, 1973 & $1 / 12$ & $11 / 12$ & & & $x$ & \\
\hline Siamese & & & & $13 / 13$ & $x$ & & \\
\hline Siamese & Elekessy et al., 1973 & & $6 / 6$ & & & & $x$ \\
\hline Siamese & Guillery et al., 1974 & & $3 / 3$ & & & & $x$ \\
\hline Siamese & Guillery and Casagrande, 1977 & & $1 / 1$ & & $x$ & & \\
\hline Siamese & & $1 / 9$ & $8 / 9$ & & & $x$ & \\
\hline Siamese & Shatz, 1977 & $11 / 12$ & $1 / 12$ & & $x$ & & \\
\hline Siamese & Montero and Guillery, 1978 & & $4 / 4$ & & $x$ & & \\
\hline Siamese & Shatz and LeVay, 1979 & $6 / 6$ & & & & $x$ & \\
\hline Siamese & Chino et al., 1980 & & $12 / 12$ & & $\mathrm{x}$ & & \\
\hline Siamese & Cooper and Blasdel, $1980^{\circ}$ & $4 / 4$ & $4 / 4$ & & $x$ & & \\
\hline Siamese & Antonini et al., 1981 & $9 / 9$ & & & $x$ & & \\
\hline Siamese & Toyama et al., 1991 & $9 / 9$ & & & $x$ & & \\
\hline Siamese & Berman and Grant, 1992 & $6 / 6$ & & & $x$ & & \\
\hline Siamese & Ault et al., 1995 & $1 / 1$ & & & & & \\
\hline hz Albino & & $1 / 1$ & & & & & \\
\hline \multirow[t]{2}{*}{ Albino } & Leventhal and Creel, 1985 & & $44 \% S$ & $56 \% S$ & $x$ & & \\
\hline & & & & $3 / 3 x$ & $x$ & & \\
\hline Albino & Schmolesky et al., 2000 & & $2 / 2 S$ & $2 / 2 x$ & $x$ & & \\
\hline hz Albinism & Leventhal et al., 1985 & $5 / 5 x$ & $5 / 5 S$ & & $x$ & & \\
\hline \multicolumn{8}{|l|}{ Mink } \\
\hline Hypopimented & Guillery et al., 1979 & $3 / 18$ & $15 / 18$ & & & $x$ & \\
\hline \multicolumn{8}{|l|}{ Ferret } \\
\hline Albino & Huang and Guillery, 1985 & $4 / 8$ & $4 / 8$ & & & $\mathrm{x}$ & \\
\hline \multirow[t]{2}{*}{ Albino } & Ackerman et al., $2003^{b}$ & $22 / 25$ & $3 / 25$ & & $x$ & & \\
\hline & & $11 / 14$ & $3 / 14$ & & & $x$ & \\
\hline Albino & Garipis and Hoffmann, 2003 & & $5 / 5$ & & & & $x$ \\
\hline \multicolumn{8}{|l|}{ Monkey } \\
\hline Albino & Guillery et al., 1984 & & & $1 / 1$ & $x$ & & \\
\hline
\end{tabular}

Cortical patterns: B, Boston; M, Midwestern; A, true albino. Cortical area (S, striate; $\mathrm{X}$, extrastriate) is indicated if study reports differential organization in different cortical areas. Method: E, single cell electrophysiology; H, histology (HRP, retrograde degeneration, ${ }^{3} \mathrm{H}$-Proline, fluorescent beads); $\mathrm{B}$, behavior; $\mathrm{hz}$, heterozogous for albinism.

${ }^{a}$ Boston and Midwestern patterns were dominant in the lower visual field and along the horizontal meridian, respectively.

${ }^{b}$ Boston and Midwestern patterns were dominant in the upper and lower visual field, respectively. The values of 22/25 and 11/14 are the lower limits of the estimated prevalence of the Boston pattern, because the upper hemifields were not tested in each of the animals. 

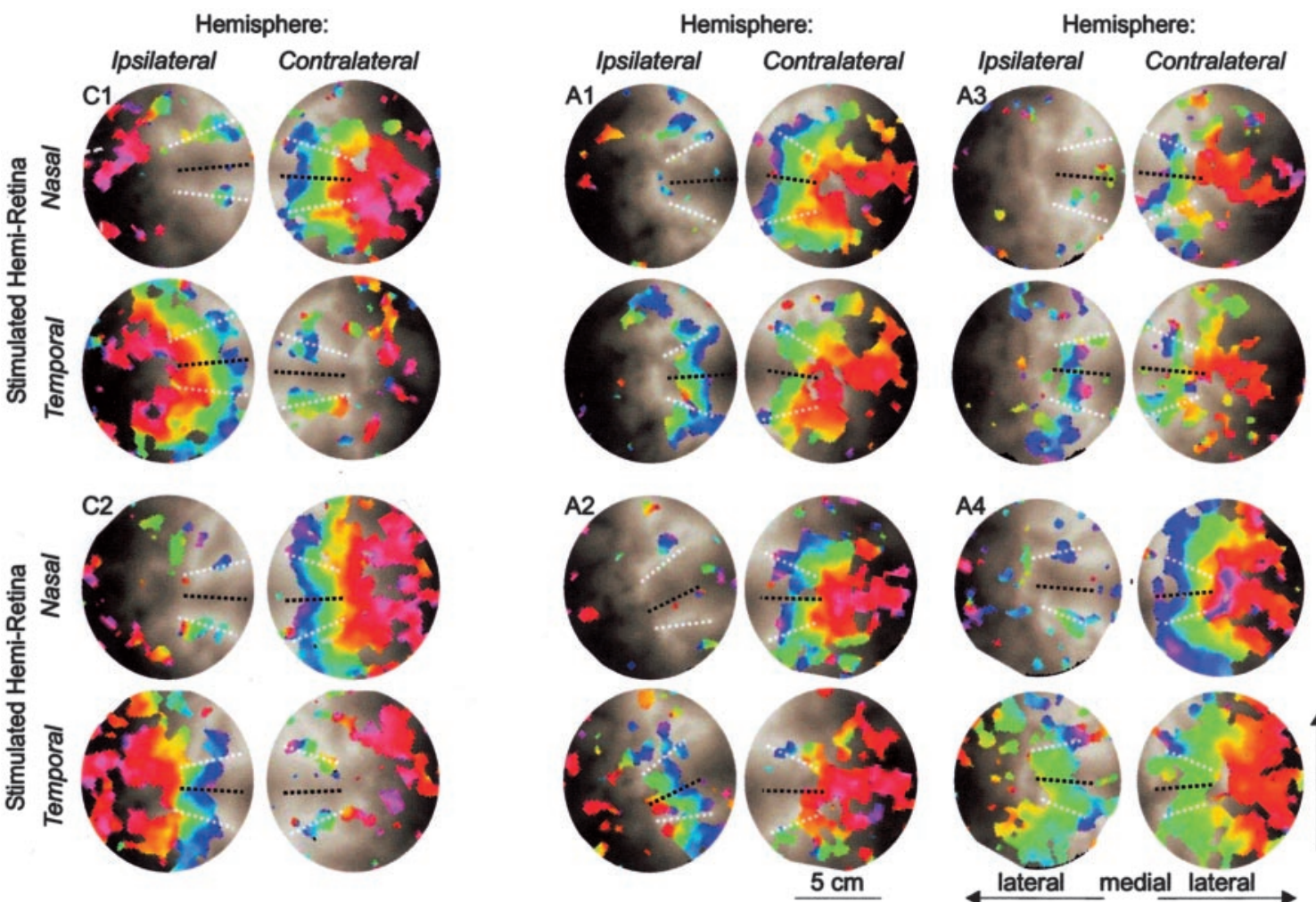

\section{Colour Key}

Stimulated

Hemi-Retina:
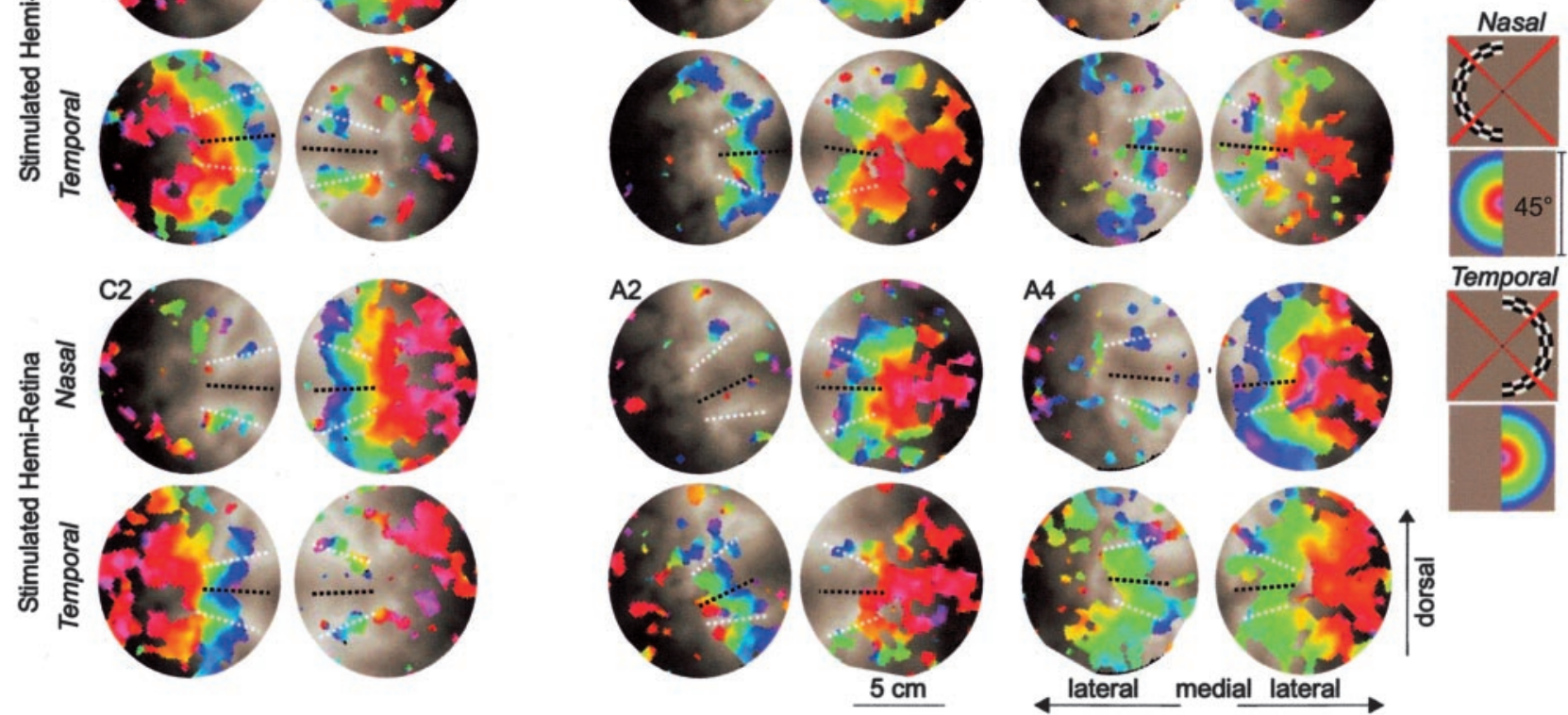

Figure 1. Mapping of the representation of visual field eccentricities on the occipital cortex in two control ( $C 1$ and $C 2$, to the left) and four albino (A1-4, to the right) subjects. Stimulation was through one eye; this and subsequent figures are drawn as if stimulation was through the left eye. For each subject, cortical activity (correlation coefficient $>0.20$ ) is plotted in false color on the flattened representations of the left and right occipital lobes. The false color indicates the phase of the cortical response and therefore the eccentricity to which that part of the cortex responds. A color key indicating eccentricity and schematics of the stimuli are given at the right. The first and third row represent the responses after stimulation of the nasal retina, whereas the second and forth row represent the responses after stimulation of the temporal retina. The upper and lower bank (white dashed lines) and the fundus (black dashed lines) of the calcarine sulcus are highlighted as cortical landmarks delimiting primary visual cortex. In the control subjects the activity is dominant on the hemisphere contralateral to the stimulated hemifield. In the albino, the activity is dominant on the hemisphere contralateral to the stimulated eye for stimulation of both nasal and temporal retina. Although this albino misrouting can be found for the central visual field, the projection reverts to the normal pattern for the eccentric representation (see Results for details).

et al., 1997), a section of this contrast-reversing checkerboard stimulus was used to stimulate monocularly either the nasal or the temporal retina in separate experiments. Seven $36 \mathrm{sec}$ cycles of the stimulus stepping either through the polar angles as a rotating wedge for polar angle mapping or through the eccentricities as an expanding ring for eccentricity mapping were presented on a liquid crystal display screen (NEC LCD2010) and viewed through a custom-built telescope. The wedge was six segments $\left(90^{\circ}\right)$ wide (see Fig. 3 ), and it stepped by $15^{\circ}$ (one segment) 12 times in each of the $36 \mathrm{sec}$ cycles. The expanding ring was three checks (5.7 $7^{\circ}$ of visual angle) wide (see Fig. 1), and it made steps of $0.95^{\circ}$ (width of half a check) 24 times in each of the $36 \mathrm{sec}$ cycles. The section of the checkerboard pattern was chosen such that it always comprised full checks, although each step was only half a check wide. For each subject we collected two data sets for eccentricity and polar angle mapping, except for A4, from whom we collected three data sets for eccentricity mapping. These data sets were averaged across repetitions in the subsequent analysis (see below).

$f M R I$ acquisition. T2* MR-images were acquired during visual stimulation using a Siemens Magnetom Vision 1.5T MRI system fitted with EPI gradient overdrive. A Multislice two-dimensional gradient echo EPI sequence [echo time (TE) $54 \mathrm{msec} ; 128 \times 128$ matrix; $240 \mathrm{~mm}$ field of view; interleaved slice order with no gap] was used to measure the blood oxygenation level-dependent (BOLD) signal as a function of time. Every $3 \mathrm{sec}, 124$-mm-thick slices were acquired perpendicular to the calcarine sulcus in a $128 \times 128$ grid covering a field of view of $240 \times 240$ (voxel size: $1.82 \times 1.82 \times 4 \mathrm{~mm}$ ) for a duration of $252 \mathrm{sec}$ (seven stimulus cycles of $36 \mathrm{sec}$ each), yielding 84 temporal samples.

Cortical flattening and $f M R I$ analysis. T1-weighted MR images (voxel size: $0.98 \times 0.98 \times 1 \mathrm{~mm}$ ) were used to create a flattened representation of the cortical gray matter (Teo et al., 1997; Wandell et al., 2000). After registration of the $\mathrm{T} 2^{\star}$-weighted images to the coordinate frame of the T1-weighted images, the fMRI time series (TS) was projected onto the flattened representation (Engel et al., 1997). The TS of each voxel underwent the following analysis: (1) time series of repeated experiments were averaged; (2) the linear trend over the 84 temporal samples was removed; (3) the first cycle of stimulation (12 temporal samples) was discarded from analysis to avoid transient onset artifacts associated with magnetization not reaching a steady state; (4) the TS was divided by the mean intensity of the voxel; (5) Fourier analysis was applied to obtain the amplitude and phase for each frequency; and (6) the correlation (c) with a sinusoid with a frequency equal to that of the visual stimulation (1/36 $\mathrm{Hz}$ ) was calculated (Engel et al., 1997). The correlation coefficients and phase values in the flattened representation were blurred by convolving a Gaussian kernel (size: $6 \times 6 \mathrm{~mm}$; half-width: $2 \mathrm{~mm}$ ) with the complex vector representation of the BOLD response. The blurred phase values were then plotted on the flattened representation of the occipital lobe in false color.

\section{Results}

\section{Cortical representation of eccentricity}

Monocular stimulation with expanding rings that stepped through increasing visual angle resulted in visual field mappings that revealed the retinotopic representation of eccentricity in occipital cortex of the normal persons (Fig. 1, subjects C1 and C2). Note that this and subsequent figures are drawn as if the observers used their left eyes in the experiments. Stimulation of the nasal and temporal retina separately resulted in retinotopic maps in the 
hemisphere contralateral and ipsilateral to the eye, respectively. A bilateral representation of the vertical meridians and fovea (Fig. 1, red/purple color code) can also be observed in the normal data, a feature that has been reported previously (Tootell et al., 1998).

In the four subjects with albinism, stimulation of the nasal retina yielded results very similar to those of the normal subjects with a smooth progression from the representation of central to peripheral visual field in the contralateral hemisphere (Fig. 1, rows 1 and 3, subjects A1-4). On stimulation of the temporal retina, however, an abnormal retinotopic representation of eccentricity was observed on the hemisphere contralateral to the stimulated eye (Fig. 1, rows 2 and 4, subjects A1-4). Our data demonstrate, therefore, the abnormal crossing of the visual projection that is typical of albinism. The regions of cortex that were activated by stimulation of the temporal retina coincided to a large extent with those activated by stimulation of the nasal retina. Moreover, the phase of the responses to nasal and temporal stimulation was similar for a given region of cortex, a feature of the data that is analyzed more formally below. The similarity of the response phases implies a mirrored repre-

sentation of nasal and temporal retina; that is, a voxel responds to stimulation of two regions of retina, one in nasal retina and another from a position in temporal retina at equal eccentricity.

Stimulation of the temporal retina at more eccentric visual angles elicited activity (Fig. 1, green, cyan, and blue color code) in the cortical hemisphere ipsilateral to the eye in the albino subjects, just as in the normal subjects. For these more eccentric field locations, therefore, temporal retinal fibers revert to the normal uncrossed projection. The topographical arrangement of the visual field in the hemisphere ipsilateral to the stimulated eye is consistent with the normal mapping, and it represents chiefly those eccentricities that were not found in the abnormally crossed representation in the contralateral hemisphere.

It should be noted that eye movements (such as the nystagmus present in subjects with albinism) would not give rise to the patterns of activity that we observed in the subjects with albinism. Eye movements could shift responses from one hemisphere to the other, but under no circumstances could the representation of eccentricity be reversed to the mirror symmetrical representation that we observe. We also monitored eye movements outside the scanner and report nystagmus of small amplitude $\left(1.5-3^{\circ}\right)$, which has been demonstrated previously to have little effect on the fMRI response (Baseler et al., 2002).

The retinotopic representations of the nasal and temporal retina in the albino cortex contralateral to the stimulated eye display qualitative differences. In the representation of the nasal retina, eccentricity is mapped to the same extent for the fundus and upper and lower banks of calcarine cortex. In contrast, the temporal retina is represented over a smaller range of eccentricities along the fundus than it is on the upper and lower banks of the calcarine cortex. The pattern of visuotopy that we reveal provides evidence for a shift of the line of decussation in albino subjects right).
Control
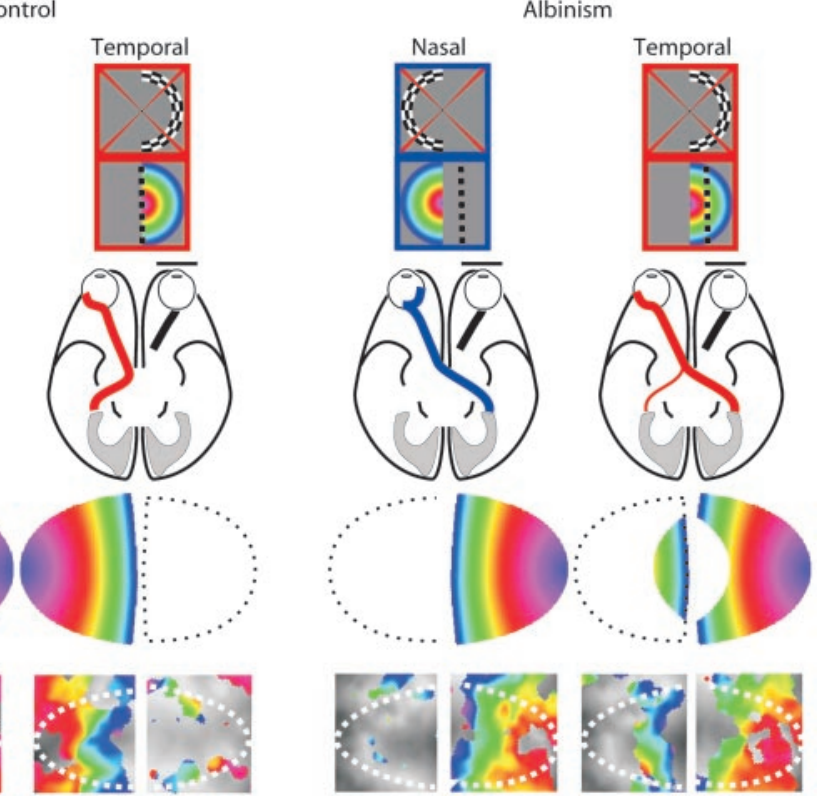

Albinism
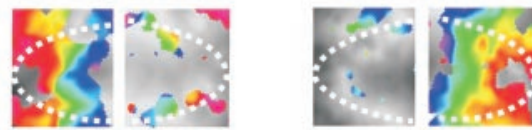

.

Figure 2. Schematic of the projection of the temporal and nasal retina of one eye in a normal control (left) and a subject with ism (right). In the top row schematics of the stimuli are given; the corresponding false color map of the eccentricities in the

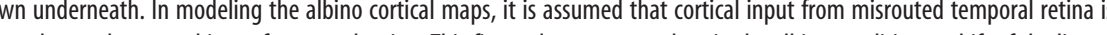
decussation into the temporal retina results in a cortical pattern similar to that which has been demonstrated in albino A1 (bottom

from the vertical meridian that passes through the fovea to a line parallel to that meridian in temporal retina. This feature is illustrated in Figure 2, which depicts the cortical representation of an eccentricity map for a control subject and a subject with albinism as derived from a model (Schwartz, 1977; von Berg, 1997) and as observed in the data (Fig. 1). For the model it is assumed that, in the control subject, the line of decussation coincides with the vertical meridian through the fovea, whereas it coincides with the vertical meridian shifted into temporal retina in the albino subject. Another assumption is that the abnormal representation of the ipsilateral visual field in the albino subject is mirrored onto the normal cortical representation, a feature that has been pointed out above and will be analyzed more formally below. Finally, the model does not take into account any bilateral representation of the visual field that may occur as a result of interhemispheric colossal connections. For the temporal retina of an albino subject, such a model produces a pattern of retinotopy of the cortical representation of the visual field with the following features. The temporal retina is represented in two complementary orderly eccentricity maps on the hemisphere ipsilateral and contralateral to the stimulated eye. The abnormal representation on the contralateral hemisphere covers the entire eccentricity range along the vertical meridian, whereas it covers only the central eccentricities along the horizontal meridian. As a result there is a notch in the abnormal cortical representation of the temporal retina. The signals that are absent in the contralateral hemisphere are represented on the ipsilateral hemisphere. This modeled pattern of retinotopy is qualitatively consistent with the data that we obtained in the calcarine cortex of the albinos as illustrated by the example of A1 in Figure 2. It should be noted, however, that the model suggests that the line of decussation is a sharp boundary, whereas the data suggest a more gradual transition. 
Although each of the albino subjects displayed very similar general features in their visuotopic maps, there were differences expressed in terms of the extent to which retinal fibers projected aberrantly (Fig. 1). We have assessed the extent of the aberrant projection quantitatively by calculating the limit of eccentricity within which $95 \%$ of voxels $(c>0.20)$ respond for two regions of interest that cover (1) the fundus of the calcarine fissure and (2) its upper and lower banks combined. This procedure was applied to data derived from stimulation with expanding rings of the nasal and temporal retina in the hemisphere contralateral to the stimulated eye. The results (Table 2) indicate that the cortical representation of the nasal retina extends over the same eccentricity in the fundus and on the banks of the calcarine for control subjects and those with albinism. This feature is entirely consistent with the normal crossed projection from nasal retina to visual cortex. For the temporal retina, eccentricity is also mapped extensively along the banks of the calcarine fissure for all subjects. In normal subjects, this mapping is consistent with the bilateral representation of the vertical meridian (Tootell et al., 1998). The extent of temporal retina mapping along the fundus of the calcarine fissure (the horizontal meridian) is a measure of the position of the visual decussation. Temporal retina is not mapped extensively along the fundus of the calcarine fissure in normal subjects, but it is in the subjects with albinism. Note, however, that the extent of the eccentricity map along the fundus of the calcarine fissure in the albinos is not as great as along the upper and lower banks. This is consistent with the displacement of the line of decussation from the vertical meridian through the fovea to a line parallel to it in temporal retina. The extent of the shift of the line of decussation into temporal retina also varied among subjects. It should be noted, however, that subjects with different nystagmus amplitudes (A1, A3, and A4) had very similar shifts in the line of decussation, indicating that there is not a strong relationship between ocular instability and the number of abnormally projecting fibers in albinism.

\section{Cortical representation of polar angle}

We examined further the retinotopic representations in the occipital lobe by mapping responses to stimuli that stepped systematically through polar angle in the normal and albino subjects. Stimulation of the nasal retina of the normal subjects resulted in a systematic progression of response phase in the contralateral hemisphere, with the representation of the horizontal meridian centered on the calcarine sulcus (Fig. 3, yellow color code, subjects $\mathrm{C} 1$ and $\mathrm{C} 2$ ). This mapping corresponds to the primary visual cortex, V1. Surrounding V1 there are further contiguous retinotopic mappings of the upper and lower field quadrants, which correspond to ventral and dorsal extrastriate visual areas, respectively. The borders of these areas are shown by reversal of the sequence of polar angles (reversal of the color codes; e.g., the ventral V1/V2 boundary is given by a red-blue-red sequence).
Stimulation of the nasal retina in the albino subjects resulted in activity in the hemisphere contralateral to the stimulated eye, with visual field mappings (Fig. 3) that expressed features similar to those of the normal; however, stimulation of the temporal retina of the albino subjects also resulted in activity in the hemisphere contralateral to the stimulated eye. The topographic representation of this abnormal visual input was found to exhibit features similar to those found in the visual field mappings of the normal crossed projection. Moreover, the visual field mappings of the normally and abnormally crossed visual inputs appear to be superimposed (Fig. 3), as they were when eccentricity was mapped (Fig. 1). This feature will be examined below in more detail. Identification of striate and extrastriate visual areas in A1 and A4 was straightforward but less so for the other subjects. It should be noted, however, that cortical mappings for the other albinos showed robust activations that are consistent with representations of the horizontal and vertical meridians, allowing V1 to be readily identified in all of the four albinos. The representations of upper and lower field quadrants were difficult to differentiate into separate extrastriate visual areas (V2, V3, etc.) in some albinos, so for any subsequent analysis the contiguous representations of the upper and lower quadrants exclusive of V1 are pooled together. Finally, it should be noted that the visual area boundaries of the albino subjects in general are more salient for the cortical representation of the nasal than of the temporal retina.

\section{Superimposed cortical representations of nasal and temporal retina}

To examine quantitatively the topography of the visual cortical representation in albinism, we followed the approach of previous studies (Hubel and Wiesel, 1971; Kaas and Guillery, 1973; Leventhal and Creel, 1985) that investigated the receptive field locations of cortex near to the V1/V2 boundary. By analogy, we investigated the cortical response along the V1/V2 boundary to stimuli that moved through polar angle. This approach serves two purposes. First, it is at the V1/V2 boundary that the different cortical patterns of visuotopy in albinism are best differentiated. Second, it helps us to assess whether V1 is organized within similar boundaries for the representations of both nasal and temporal retina. We calculated the BOLD response for voxels selected on the basis of the following criteria. (1) We identified two regions of visual cortex that extended (A) dorsally and (B) ventrally, from the representation of the horizontal meridian in V1 to the next representation of the horizontal meridian in extrastriate cortex. (2) Within these spatial regions we selected only voxels that exceeded a correlation coefficient of 0.20 after stimulation of the nasal retina and responded to visual field locations that were adjacent $\left( \pm 30^{\circ}\right.$ of polar angle $)$ to either the upper or lower vertical meridians. This process of selection highlights only the voxels at the V1/V2 boundary with robust activation to the upper and

\begin{tabular}{|c|c|c|c|c|c|c|c|}
\hline & & \multicolumn{6}{|c|}{ Controls $(n=4)$} \\
\hline & & Median & Range & A1 & $\mathrm{A} 2$ & A3 & A4 \\
\hline \multirow[t]{2}{*}{ Upper and lower banks of the calcarine } & Nasal retina & 19.4 & $18.4-19.6$ & 19.7 & 20.7 & 20.9 & 21.3 \\
\hline & Temporal retina & 19.8 & $18.0-21.7$ & 19.0 & 19.8 & 19.2 & 20.3 \\
\hline \multirow[t]{2}{*}{ Fundus of the calcarine } & Nasal retina & 19.8 & $19.3-20.3$ & 20.3 & 18.8 & 16.6 & 20.3 \\
\hline & Temporal retina & 1.0 & $0-4.1$ & 13.9 & 5.5 & 11.9 & 13.7 \\
\hline
\end{tabular}


St imulated Hemiretina:

Nasal

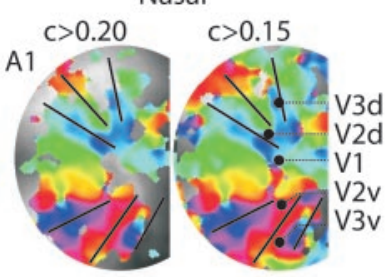

Temporal
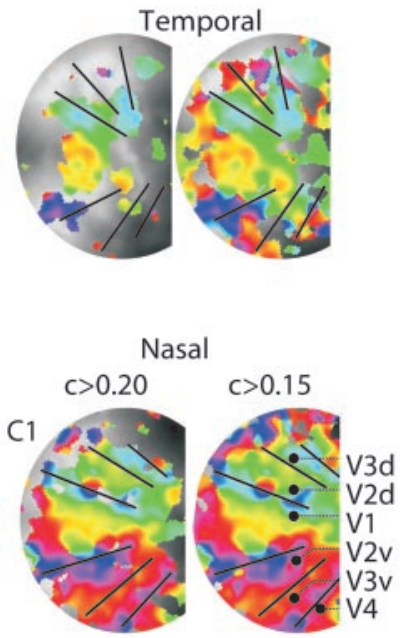

Stimulated Hemiretina: Nasal
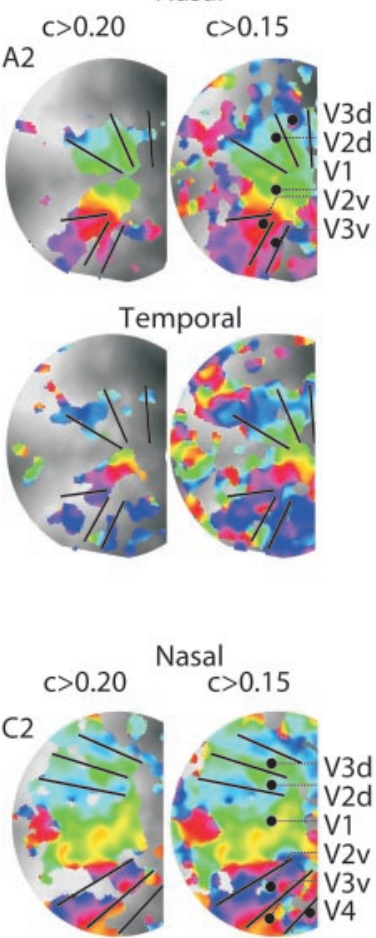

Nasal

c $>0.15$

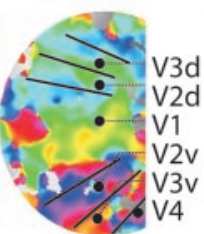

Stimulated Hemiretina: Nasal

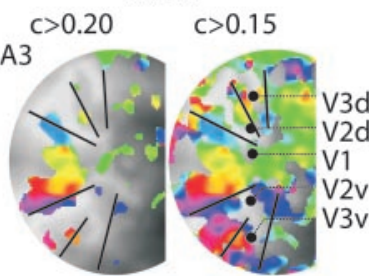

Temporal

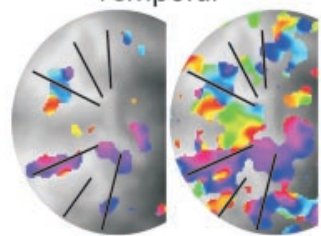

Color Key

Stimulated Hemiretina:

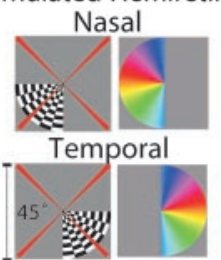

St imulated Hemiretina:

Nasal
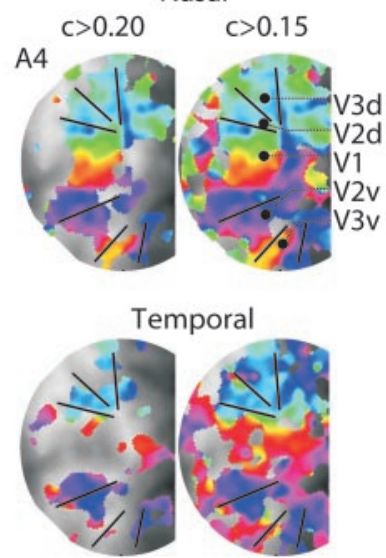

Figure 3. Mapping of the representation of the polar angles in the visual field on the occipital cortex contralateral to the stimulated eye in two control subjects (C1 and $\mathrm{C2}$, bottom row) after stimulation of the nasal retina and in four albino subjects ( $\mathrm{A} 1-4$ ) after stimulation of the nasal (top row) and temporal (middle row) retina. Cortical activity $(c>0.20$ and $c>0.15$ ) is plotted in false color on flattened representations of the right occipital lobes. The false color indicates the phase of the cortical response and therefore the polar angle to which that part of the cortex responds. A color key indicating polar angle and schematics of the stimuli is given at the bottom right. The positions of the reversals of the phase progressions on the flattened representations allow visual area boundaries to be identified. These boundaries were determined after stimulation of the nasal retina of the left eye (black lines). There is a correspondence of these boundaries, especially in V1, to those obtained after stimulation of the temporal retina of the albino subjects.

lower vertical meridians of the nasal retina. The BOLD response of these voxels is shown in Figure 4 (light trace) as averaged time series, each point being the average of 12 stimulation blocks (two runs of 6 blocks). The key question, however, is what response is elicited from these same voxels during stimulation of the temporal retina. Figure 4 (solid traces) shows that only the albino subjects exhibited robust signals when the temporal retina was stimulated. In fact, the BOLD signals at the V1/V2 boundary elicited by innervation via the normally and abnormally crossed visual pathways in albinos are very similar in amplitude. Moreover, the mean BOLD response appears to have the same phase for stimulation of nasal and temporal retina; so the voxels that are sensitive to stimulation near the vertical meridian of nasal retina also display sensitivity to locations in temporal retina that mirror the nasal retinal locations.

The phase of the BOLD signal indicates the retinal location (eccentricity or polar angle) to which a voxel is sensitive. In our experiments that separately stimulated the two hemi-retinas, there will be two phase measurements that reflect locations in nasal and temporal retina, respectively. Thus, a voxel that displays the same response phase during stimulation of the nasal and temporal retina possesses a representation of one location in nasal retina and another location in temporal retina that exactly mirrors it. The phase of the response, therefore, reveals the "receptive field" locations of a voxel in nasal and temporal retina. The extent to which maps of nasal and temporal retina are superimposed in cortex can be assessed quantitatively by calculating the difference between the response phases (receptive field loca- tions) obtained during the two stimulation conditions. We calculated the separations in receptive field locations for voxels in striate and extrastriate areas for stimulation of nasal and temporal retina and then determined a normalized frequency distribution of the separations. The voxels selected for this analysis were those on the occipital lobe contralateral to the stimulated eye and for which correlation exceeded 0.20 for stimulation of the temporal retina in subjects with albinism. Therefore, we apply selection criteria to identify the voxels that display robust activity through abnormally crossed innervation of the cortex in albinism and evaluate their responses when innervated by the normally crossed pathways. In normal subjects we made a comparison between two experiments in which the same hemi-retina was stimulated to determine what frequency distribution reflects "perfect" overlap of visuotopic maps.

An example of the distributions is given for eccentricity mapping in V1 in Figure $5 A$. For normal subjects, the profile of this plot reveals that if two identical stimulation conditions are considered, the greatest proportion of voxels has receptive fields that are separated by small eccentricities, whereas the smallest proportion of voxels has receptive fields separated by large eccentricities. Stimulation of the nasal and temporal retina in normal subjects reveals a very different result, in which the profile of the plot is far shallower. Interestingly, the highest proportion of voxels still has small differences between receptive field locations, and this probably reflects the bilateral representation of retinal locations near the vertical meridian and fovea (Fig. 1). For the albino subjects, stimulation of the nasal and temporal retina yields dis- 


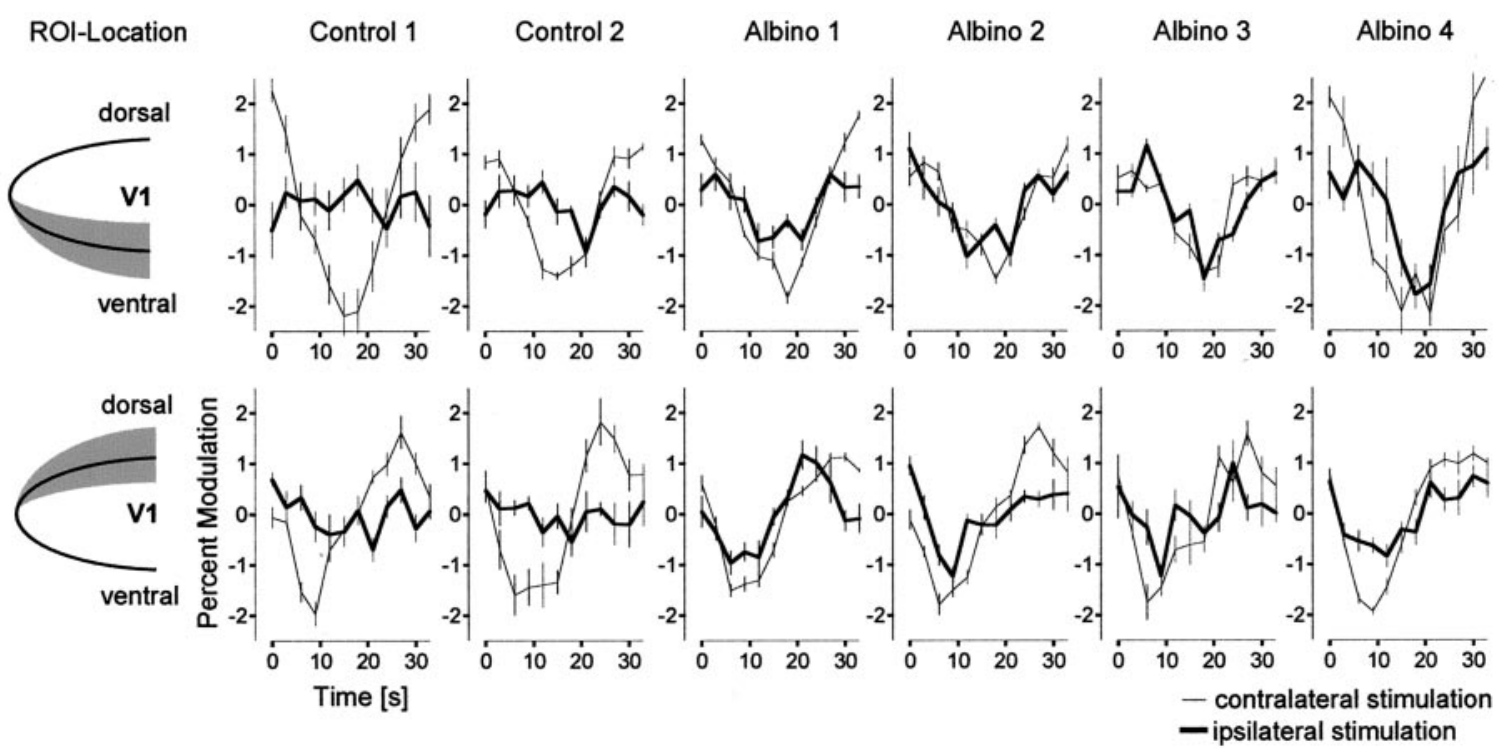

Figure 4. BOLD signal (mean of $2 \times 6$ cycles \pm SEM) on the right hemisphere after stimulation of the nasal and temporal retina of the left eye. ROl for traces in left and right column cover lower and upper bank of calcarine sulcus, respectively. For details of ROI definition see Results. In albinos and controls, stimulation of the nasal retina results in a strong response (thin traces), which differs in phase for the lower and upper bank of the calcarine (top and bottom row, respectively). After stimulation of the temporal retina, this activity is strongly reduced in the control subjects, whereas it is still prominent in the subjects with albinism (thick traces in each panel).

tributions very different from those obtained from the normal subjects. In fact, for two of the albino subjects (A1 and A3) the distribution is very similar to the one derived from normal subjects during stimulation of common retinal regions. In V1, therefore, the representations of the nasal and temporal retina appear to overlap each other in the albinos. This overlap is not perfect, however, particularly for A2 and A4. We have characterized the relative frequency distributions with a single metric, the median, which is shown for the different groups and conditions in Table 3. The data, when compared statistically, reveal that the median separation of voxel receptive fields does not systematically differ between the group of albinos and the normal subjects, when two identical stimulation conditions are compared in the normal subjects. In contrast, the distribution of medians in the albino group is generally different from the normal data derived from stimulation of nasal and temporal retina. One exception exists for striate responses to stimuli moving through polar angle. It should also be noted that the analysis that we present in Table 3 takes phase wrapping into account. Because the cyclical stimulus presents the most central (minimum phase) and eccentric visual field (maximum phase) locations in close temporal proximity, small amounts of uncertainty in determining absolute phase can produce great phase differences, where the actual differences in receptive field location are small. This problem can either be ignored, leading to an overestimate of receptive field separation, or addressed (as in Table 3 ) by the assumption that large phase differences are caused by phase wrap, leading to an underestimate of receptive field separation. We found that the median increased very little $(1-10 \%)$ and that all of the statistical comparisons remained the same as presented in Table 3, when we did not account for phase wrapping.

A frequency distribution of the separations in receptive field locations in nasal and temporal retina can also be compared with similar data constructed from electrophysiological studies of visuotopy in other albino animals. In electrophysiological studies, receptive field locations are obtained for a series of cells along electrode penetrations. Cells can be grouped into zones of those
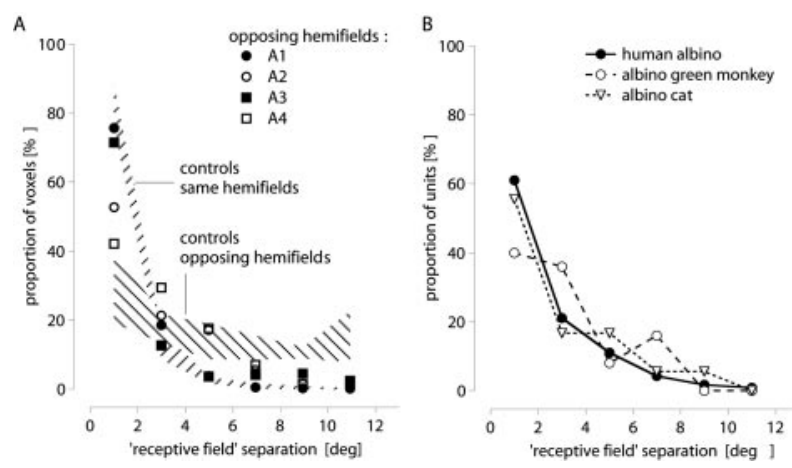

Figure 5. A, Frequency distributions of the separations of nasal and temporal retinal coordinates of voxel receptive field locations (see Results for details). Data are given for voxels in V1 for four albino subjects as indicated in the legend. A range for the control distributions is given ( $\pm 1 S D, 4$ hemispheres) for two different comparisons: one when stimulation was repeated in the same hemi-retina and the other for stimulation of different hemi-retinas. $B$, Cross-study comparison of the mirror-symmetrical superposition of the cortical representation of the normal input from nasal retina and of the abnormal input from temporal retina in humans (number of subjects, $n=4$; number of units, $m=679)$, monkey $(n=1 ; m=30)$, and cats with albinism $(n=2 ; m=18)$. The proportion of units as a function of the eccentricity difference (receptive field separation) between the two maps is plotted according to Figure $5 \mathrm{~A}$.

that respond to the nasal retina and those that respond to temporal retina. The precision with which the neighboring representations of nasal and temporal retina mirror each other can be calculated by taking the difference in the receptive-field locations of cells that abut the boundary between zones representing nasal and temporal retina. We have made these calculations for two studies, one of an albino monkey (Guillery et al., 1984) and the other of albino cats (Leventhal and Creel, 1985). The cells that we have examined had receptive fields that were within the same region of visual space as we stimulated here. It should also be noted that the cortical separations between cells and the cortical dimensions of the nasal and temporal zones were significantly smaller than the voxel dimensions used here. For any one voxel, 
Table 3. Differences in voxel receptive field locations calculated when (1) the same stimulation conditions were compared in normals, (2) stimulation of opposing hemifields was compared in normals, and (3) stimulation of opposing hemifields was compared in albinos

\begin{tabular}{|c|c|c|c|c|c|c|}
\hline & & \multicolumn{3}{|c|}{ Median difference (in degrees) } & \multirow[b]{2}{*}{3 versus 1} & \multirow[b]{2}{*}{3 versus 2} \\
\hline & & 1. Control same & 2. Control opposing & 3. Albino opposing & & \\
\hline \multirow[t]{4}{*}{ Striate } & Rings & 0.8 & 4.7 & 1.5 & 0.39 & $<0.05$ \\
\hline & $0-11^{\circ}$ & & & & & \\
\hline & Wedges & 5.8 & 36.5 & 20.3 & 0.24 & 0.09 \\
\hline & $0-90^{\circ}$ & & & & & \\
\hline \multirow[t]{4}{*}{ Extrastriate } & Rings & 1.2 & 4.1 & 1.5 & 1.00 & $<0.05$ \\
\hline & $0-11^{\circ}$ & & & & & \\
\hline & Wedges & 17.2 & 65.6 & 25.5 & 0.59 & $<0.05$ \\
\hline & $0-90^{\circ}$ & & & & & \\
\hline
\end{tabular}

Data are given for group means of the median separation of receptive field locations, with values for stimulation with rings and wedges given for striate and extrastriate areas. For the eccentricity mapping, data are specified in degrees of visual angle; for polar angle mapping, data are specified in degrees of polar angle. Statistical comparisons were made between the albino group and the two control conditions with an unpaired $t$ test that was corrected for multiple comparisons (Bonferroni method) and also accounted for unequal variance in the group distributions.

therefore, we expect a number of transitions from one zone to another to contribute to the overall difference between the voxel receptive field locations. Thus, we believe that the magnitude of the shift in receptive field locations for the transition from one zone to the other in the animal studies provides a good measure to compare with our data. The relative frequency distributions are presented in Figure $5 B$ and show very similar features across the three studies. The principal finding from all three studies is that the mismatch between receptive field locations in nasal and temporal retina for a given cortical location is small $\left(\sim 1.5^{\circ}\right)$.

\section{Relative strength of cortical representation of inputs from nasal and temporal retina}

Our fMRI data give us the opportunity to examine the relative strength of the crossed and uncrossed inputs to visual cortex (Fig. 6). We consider here that the amplitude of the BOLD response is indicative of the strength of the signals elicited by the stimulation of nasal and temporal retinal inputs. We defined two regions of interest (ROI), one representing striate and the other representing extrastriate visual areas. The ROI definition is on the basis of the voxels that exceeded a correlation coefficient of 0.20 during stimulation of both nasal and temporal retina. Thus striate and extrastriate visual areas that are driven by both the normal and the abnormal projection are identified. We then calculated the BOLD response as the mean of the response amplitude of each voxel after stimulation of both temporal and nasal retina. This value was obtained for each ROI and hence for striate and extra-
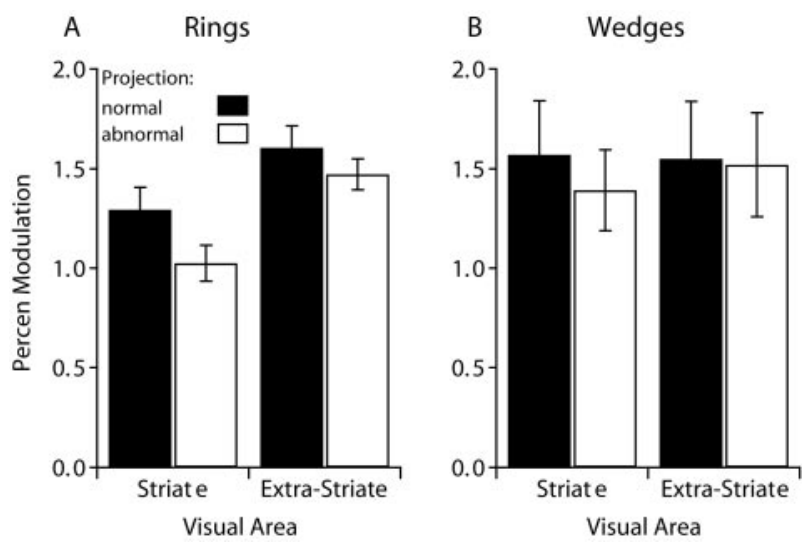

Figure 6. The mean fMRI signal amplitude after nasal (normal projection, filled bars) and temporal retinal stimulation (abnormal projection, open bars). The average response strength of the four albino subjects is depicted (mean \pm 1 SD). Data are given for striate cortex and for extrastriate cortex that includes V2 and V3. striate areas. The relative activity of the abnormal and normal representation in striate and extrastriate areas allows one to assess whether the abnormal representation compared with the normal representation is suppressed when being passed on to extrastriate areas. We observed a small but significant reduction of the activity in the abnormal compared with the normal representation (average reduction: $10 \%$; repeated measures ANOVA; $p=$ 0.0275 ); however, the extrastriate activation is no further reduced than the striate activation (reduction of abnormal representation: 16 and $3 \%$ in striate and extrastriate cortex, respectively). There is no quantitative manner in which we can compare the relative strength of striate responses in the previous studies of other albino animals. It is important to note, however, that Guillery et al. (1984) indicated a very small difference between the number of cells that responded to nasal and temporal retina in striate and extrastriate cortex of the monkey.

In showing that abnormally crossing input drives extrastriate areas, we rule out the possibility that the striate cortical signals that we have measured result from subthreshold potentials that fMRI may be sensitive to (Logothetis et al., 2001). Thus, the observed extrastriate activity shows that there must have been overt striate activity and that the abnormal input to the cortex cannot have been suppressed completely as would be expected from a cortical organization that follows the Midwestern pattern, which has been observed previously in animal models [Table 1, Kaas and Guillery (1973)].

\section{Discussion}

\section{Striate cortex}

Previous evoked potential (Coleman et al., 1979; Apkarian et al., 1983; Morland et al., 2002) and fMRI (Hedera et al., 1994; Morland et al., 2001) studies have revealed correlates of the abnormal routing of retinal afferents in human albinism; however, the visuotopic map within the cortex of human albinos has not been explicitly investigated before. Our measurements demonstrate clearly that the abnormally crossing fibers from the temporal retina of the albino form a mirror-image map in striate cortex that is superimposed on the normal retinotopic map of the nasal retina. Voxels in V1, therefore, represent two visual field locations that are mirrored about the vertical meridian. This finding is consistent with the mapping reported for the only other albino primate to be studied, Cercopithecus aethiops (Guillery et al., 1984). Our results, taken together with those of Guillery et al. (1984), indicate that the profound reorganization of the thalamocortical connections that has been reported in other hypopigmented and albino mammals [Table 1, Hubel and Wiesel (1971)] is absent in the albino primate. 
The nature of our data acquisition has allowed us to recruit information across a large contiguous region of the occipital cortex. We can conclude, therefore, that the superposition of the mappings is found across the total striate representation of the abnormal cortical input in human albinism. In electrophysiological studies, similar conclusions can only be drawn by extrapolating a limited, albeit high resolution, sampling of striate cortex (Guillery et al., 1984; Leventhal and Creel, 1985; Schmolesky et al., 2000). There is evidence that such extrapolations may need cautious interpretation because different visuotopic patterns (Midwestern and Boston) have been found in different regions of striate cortex within the same individual [Table 1, Cooper and Blasdel (1980); Ackerman et al. (2003)]. The strength of this study, therefore, lies in the extensive area of cortex that is surveyed.

\section{Extrastriate cortex}

Our measurements have also allowed us to investigate the topographic mapping of the visual field in extrastriate visual areas. Very little information concerning the extrastriate representations of the normal and abnormal albino projections exist: the only data originate from area 18 , where it meets area 17 . Some of this work [Table 1, Leventhal and Creel (1985); Leventhal et al. (1985); Schmolesky et al. (2000)], however, revealed that some hypopigmented and albino animals appear to display different visuotopic representations in areas 17 (Midwestern) and 18 (true albino). In contrast, the visuotopic map in area 18 of the single primate studied was the same as that expressed in area 17 (Guillery et al., 1984). Our data confirm the previous work in the primate by documenting a pattern of superimposed representations of nasal and temporal retina in extrastriate areas, the same pattern as in striate cortex. Our measurements extend previous work by recording from cortex dorsal and ventral of striate cortex to cover $\mathrm{V} 2$ and $\mathrm{V} 3$, visual areas that have not been characterized previously.

\section{Suppression}

There is strong evidence that input from abnormally routed fibers from temporal retina can be substantially suppressed in primary visual cortex of hypopigmented mammals (Hubel and Wiesel, 1971; Kaas and Guillery, 1973; Shatz, 1977; Huang and Guillery, 1985). Our measurements indicate that the abnormal input from temporal retina resulted in levels of activity that were suppressed little compared with those elicited by normal input from nasal retina. This was true for both striate and extrastriate visual areas and rules out the presence of marked or even complete intracortical suppression of the aberrant cortical input that is observed in other species. A previous study on a single primate indicates only a small reduction in the number of cells that are responsive to temporal compared with nasal retina, a finding that our results support (Guillery et al., 1984).

\section{Line of decussation}

In the hypopigmented cat, ferret, and monkey, the line in the retina that divides fibers that project ipsilaterally from those that project contralaterally is shifted into temporal retina. Therefore, temporal retina representing the most peripheral visual field tends to revert to a normal ipsilateral projection (Guillery et al., 1984; Leventhal and Creel, 1985; Morgan et al., 1987). We have been able to document that in human albinism this line of decussation is also shifted temporally and that it runs parallel to the vertical meridian through the fovea. Furthermore, we revealed (Table 2) that the degree to which the line of decussation was shifted varied between subjects. In mice there is strong evidence that the extent of the misrouting of retinal afferents is proportional to the degree of retinal hypopigmentation (LaVail et al., 1978; Balkema and Drager, 1990), and a similar relation has been suggested for cats (Ault et al., 1995) and mink (Sanderson et al., 1974). It would be valuable, therefore, to determine the shift in the line of decussation for a large number of subjects with albinism and compare it with levels of retinal pigmentation and other phenotypic characteristics.

\section{Behavioral implications}

The superimposed retinotopic maps that we describe in albinism have strong implications for the relationship between intracortical organization of the visual areas and visual perception. Many cells in striate cortex integrate information from ocular dominance columns and therefore have binocular input, as do the majority of cells in extrastriate areas. The connections that normally give rise to binocular integration in the albino's visual cortex with its superimposed maps would result in cells with separated receptive fields in opposing hemifields. In animal models of albinism, such cells are extremely rare (Hubel and Wiesel, 1971; Leventhal and Creel, 1985) and have not been reported at all in the albino monkey studied (Guillery et al., 1984). Accordingly, there are no reports of a specific interaction between stimuli presented in opposing hemifields in human albinism; however, altered psychophysical performance for nasal and temporal retinal stimulation has been reported (St. John and Timney, 1981).

Additional experimentation that probes behavioral responses to stimuli presented to mirrored locations in nasal and temporal retina is needed to explore how the superposition of cortical maps may influence perception.

\section{Conclusion}

Our measurements indicate that the human albino exhibits a topographical representation of the visual field (the true albino pattern) that is rarely observed in hypopigmented mammals; however, the mapping that we report is consistent with that documented in the only other primate studied (Guillery et al., 1984). It would appear, therefore, that developmental mechanisms that allow the thalamocortical projections to be reorganized in some mammals are not available to the primate.

We observed the abnormal projection of the temporal retina predominantly in the central part of the visual field. In this respect, therefore, the fundamental origin of the albino abnormality appears to be expressed in the same way in the primate as in other species. Furthermore, we have been able to detail the line of decussation in the human as a vertical meridian that is shifted into temporal retina.

We have also been able to document that the visuotopic representation of the nasal and temporal retina is superimposed in early extrastriate areas. It is likely, therefore, that cells in striate and extrastriate areas that normally develop to respond to binocular input will not develop to recruit information from conflicting, mirror-symmetric retinal locations. Consequently, no superposition of the maps at the level of single neurons should be expected. It remains to be seen whether cells in striate or extrastriate areas that respond to nasal and temporal retina are segregated into zones (or columns) in humans as they are in the striate cortex of other albino animals. Additional fMRI experiments performed at higher resolution may be able to reveal this feature. 


\section{References}

Ackerman CJ, Tolhurst DJ, Morgan JE, Baker GE, Thompson ID (2003) The relay of visual information to the lateral geniculate nucleus and the visual cortex in albino ferrets. J Comp Neurol 20:1253-1260.

Antonini A, Berlucchi G, Di Stefano M, Marzi CA (1981) Differences in binocular interactions between cortical areas 17 and 18 and superior colliculus of Siamese cats. J Comp Neurol 200:597-611.

Apkarian P, Reits D, Spekreijse H, Van Dorp D (1983) A decisive electrophysiological test for human albinism. Electroencephalogr Clin Neurophysiol 55:513-531.

Ault SJ, Leventhal AG, Vitek DJ, Creel DJ (1995) Abnormal ipsilateral visual field representation in areas 17 and 18 of hypopigmented cats. J Comp Neurol 354:181-192.

Bach M (1996) The Freiburg Visual Acuity test: automatic measurement of visual acuity. Optom Vis Sci 73:49-53.

Balkema GW, Drager UC (1990) Origins of uncrossed retinofugal projections in normal and hypopigmented mice. Vis Neurosci 4:595-604.

Baseler HA, Brewer AA, Sharpe LT, Morland AB, Jägle H, Wandell BA (2002) Reorganization of human cortical maps caused by inherited photoreceptor abnormalities. Nat Neurosci 5:364-370.

Berman NE, Grant S (1992) Topographic organization, number, and laminar distribution of callosal cells connecting visual cortical areas 17 and 18 of normally pigmented and Siamese cats. Vis Neurosci 9:1-19.

Chino YM, Shansky MS, Pizzi WJ (1980) Receptive field properties of simple and complex striate neurons in Siamese cats. J Comp Neurol 190:63-86.

Coleman J, Sydnor CF, Wolbarsht ML, Bessler M (1979) Abnormal visual pathways in human albinos studied with visually evoked potentials. Exp Neurol 65:667-679.

Cooper ML, Blasdel GG (1980) Regional variation in the representation of the visual field in the visual cortex of the Siamese cat. J Comp Neurol 193:237-253

Creel DJ (1971) Visual system anomaly associated with albinism in the cat. Nature 231:465-466.

Creel DJ, Witkop CJ, King RA (1974) Asymmetric visually evoked potentials in human albinos: evidence for visual system anomalies. Invest Ophthalmol Vis Sci 430-440.

DeYoe EA, Carman GJ, Bandettini P, Glickman S, Wieser J, Cox R, Miller D, Neitz J (1996) Mapping striate and extrastriate visual areas in human cerebral cortex. Proc Natl Acad Sci USA 93:2382-2386.

Elekessy EI, Campion JE, Henry GH (1973) Differences between the visual fields of Siamese and common cats. Vision Res 13:2533-2543.

Engel SA, Glover GH, Wandell BA (1997) Retinotopic organization in human visual cortex and the spatial precision of functional MRI. Cereb Cortex 7:181-192.

Garipis N, Hoffmann KP (2003) Visual field defects in albino ferrets (Mustela putorius furo). Vision Res 43:793-800.

Guillery RW (1986) Neural abnormalities of albinos. Trends Neurosci 18:364-367.

Guillery RW, Casagrande VA (1977) Studies of the modifiability of the visual pathways in Midwestern Siamese cats. J Comp Neurol 174:15-46.

Guillery RW, Kaas JH (1973) Genetic abnormality of the visual pathways in a "white" tiger. Science 180:1287-1289.

Guillery RW, Casagrande VA, Oberdorfer MD (1974) Congenitally abnormal vision in Siamese cats. Nature 252:195-199.

Guillery RW, Okoro AN, Witkop CJ Jr (1975) Abnormal visual pathways in the brain of a human albino. Brain Res 96:373-377.

Guillery RW, Oberdorfer MD, Murphy EH (1979) Abnormal retinogeniculate and geniculo-cortical pathways in several genetically distinct color phases of the mink (Mustela vison). J Comp Neurol 185:623-655.

Guillery RW, Hickey TL, Kaas JH, Felleman DJ, Debruyn EJ, Sparks DL (1984) Abnormal central visual pathways in the brain of an albino green monkey (Cercopithecus aethiops). J Comp Neurol 226:165-183.

Hedera P, Lai S, Haacke EM, Lerner AJ, Hopkins AL, Lewin JS, Friedland RP (1994) Abnormal connectivity of the visual pathways in human albinos demonstrated by susceptibility-sensitized MRI. Neurology 44:1921-1926.
Huang K, Guillery RW (1985) A demonstration of two distinct geniculocortical projection patterns in albino ferrets. Brain Res 352:213-220.

Hubel DH, Wiesel TN (1971) Aberrant visual projections in the Siamese cat. J Physiol (Lond) 218:33-62.

Jeffery G (1997) The albino retina: an abnormality that provides insight into normal retinal development. Trends Neurosci 20:165-169.

Kaas JH, Guillery RW (1973) The transfer of abnormal visual field representations from the dorsal lateral geniculate nucleus to the visual cortex in Siamese cats. Brain Res 59:61-95.

LaVail JH, Nixon RA, Sidman RL (1978) Genetic control of retinal ganglion cell projections. J Comp Neurol 182:399-421.

Leventhal AG, Creel DJ (1985) Retinal projections and functional architecture of cortical areas 17 and 18 in the tyrosinase-negative albino cat. J Neurosci 5:795-807.

Leventhal AG, Vitek DJ, Creel DJ (1985) Abnormal visual pathways in normally pigmented cats that are heterozygous for albinism. Science 229:1395-1397.

Logothetis NK, Pauls J, Augath M, Trinath T, Oeltermann A (2001) Neurophysiological investigation of the basis of the fMRI signal. Nature 412:150-157.

Lund RD (1965) Uncrossed visual pathways of hooded and albino rats. Science 149:1506-1507.

Montero VM, Guillery RW (1978) Abnormalities of the cortico-geniculate pathway in Siamese cats. J Comp Neurol 179:1-12.

Morgan JE, Henderson Z, Thompson ID (1987) Retinal decussation patterns in pigmented and albino ferrets. Neuroscience 20:519-535.

Morland AB, Baseler HA, Hoffmann MB, Sharpe LT, Wandell BA (2001) Abnormal retinotopic representations in human visual cortex revealed by fMRI. Acta Psychol (Amst) 107:229-247.

Morland AB, Hoffmann MB, Neveu M, Holder GE (2002) Abnormal visual projection in a human albino studied with functional magnetic resonance imaging and visual evoked potentials. J Neurol Neurosurg Psychiatry 72:523-526.

Oetting WS, Brilliant MH, King RA (1996) The clinical spectrum of albinism in humans. Mol Med Today 2:330-335.

Sanderson KJ, Guillery RW, Shackelford RM (1974) Congenitally abnormal visual pathways in mink (Mustela vison) with reduced retinal pigment. J Comp Neurol 154:225-248.

Schmolesky MT, Wang Y, Creel DJ, Leventhal AG (2000) Abnormal retinotopic organization of the dorsal lateral geniculate nucleus of the tyrosinase-negative albino cat. J Comp Neurol 427:209-219.

Schwartz EL (1977) Spatial mapping in the primate sensory projection: analytic structure and relevance to perception. Biol Cybern 25:181-194.

Sereno MI, Dale AM, Reppas JB, Kwong KK, Belliveau JW, Brady TJ, Rosen BR, Tootell RB (1995) Borders of multiple visual areas in humans revealed by functional magnetic resonance imaging. Science 268:889-893.

Shatz C (1977) A comparison of visual pathways in Boston and Midwestern Siamese cats. J Comp Neurol 171:205-228.

Shatz CJ, LeVay S (1979) Siamese cat: altered connections of visual cortex. Science 204:328-330.

St. John R, Timney B (1981) Sensitivity deficits consistent with aberrant crossed visual pathways in human albinos. Invest Ophthalmol Vis Sci 21:873-877.

Teo PC, Sapiro G, Wandell BA (1997) Creating connected representations of cortical gray matter for functional MRI visualization. IEEE Trans Med Imaging 16:852-863.

Tootell RB, Mendola JD, Hadjikhani NK, Liu AK, Dale AM (1998) The representation of the ipsilateral visual field in human cerebral cortex. Proc Natl Acad Sci USA 95:818-824.

Toyama K, Kitaoji H, Umetani K (1991) Binocular neuronal responsiveness in Clare-Bishop cortex of Siamese cats. Exp Brain Res 86:471-482.

von Berg J (1997) A formal mapping model for striate cortex. Perception 26:17.

Wandell BA, Chial S, Backus BT (2000) Visualization and measurement of the cortical surface. J Cognit Neurosci 12:739-752. 